\title{
Use of high-resolution measurements for the retrieval of temperature and gas-concentration profiles from outgoing infrared spectra in the presence of cirrus clouds
}

\author{
Xianglei L. Huang, Yuk L. Yung, and Jack S. Margolis
}

\begin{abstract}
We explore ways in which high-spectral-resolution measurements can aid in the retrieval of atmospheric temperature and gas-concentration profiles from outgoing infrared spectra when optically thin cirrus clouds are present. Simulated outgoing spectra that contain cirrus are fitted with spectra that do not contain cirrus, and the residuals are examined. For those lines with weighting functions that peak near the same altitude as the thin cirrus, unique features are observed in the residuals. These unique features are highly sensitive to the resolution of the instrumental line shape. For thin cirrus these residual features are narrow $\left(\leq 0.1 \mathrm{~cm}^{-1}\right)$, so high spectral resolution is required for unambiguous observation. The magnitudes of these unique features are larger than the noise of modern instruments. The sensitivities of these features to cloud height and cloud optical depth are also discussed. Our sensitivity studies show that, when the errors in the estimation of temperature profiles are not large, the dominant contribution to the residuals is the misinterpretation of cirrus. An analysis that focuses on information content is also presented. An understanding of the magnitude of the effect and of its dependence on spectral resolution as well as on spectral region is important for retrieving spacecraft data and for the design of future infrared instruments for forecasting weather and monitoring greenhouse gases. (C) 2003 Optical Society of America
\end{abstract}

OCIS codes: $\quad 000.4430,010.1310,010.3920$.

\section{Introduction}

In the past two decades, atmospheric measurements from the National Oceanic and Aeronautic Administration's (NOAA's) polar orbiting satellite systems, such as measurements by the Microwave Sounding Unit (MSU) and the High-Resolution Infrared Sounder (HIRS) flown on the Television and Infrared Observation Satellite [(TIROS)-N] Operational Vertical Sounder ${ }^{1}$ (TOVS), have been assimilated into operational numerical weather prediction (NWP) models. In 1987, after evaluating the effects of the first ten years of such measurements on NWP accu-

X. L. Huang and Y. L. Yung (yly@gps.caltech.edu) are with the Division of Geological and Planetary Sciences, California Institute of Technology, Pasadena, California 91125. J. S. Margolis is with Remote Sensing Analysis Systems, Inc., Pasadena, California 91001.

Received 21 August 2002; revised manuscript received 2 January 2003.

0003-6935/03/122155-11\$15.00/0

(C) 2003 Optical Society of America racy, the World Meteorological Organization concluded that significantly improving the NWP will require making global temperature and moisture soundings with radiosonde accuracy. ${ }^{2}$ This is equivalent to obtaining temperature profiles with $1-\mathrm{K}$ accuracy in 1-km-thick layers and humidity profiles with $90 \%$ accuracy in the troposphere. To fulfill these requirements a high-spectral-resolution infrared sounder with a high signal-to-noise ratio is needed. Meanwhile, precise monitoring of the greenhouse gases and their interaction with other gases in the troposphere requires an even higherspectral-resolution infrared sounder to resolve absorption lines. As a result, we expect that in this decade several high-spectral-resolution IR sounders, such as the Atmospheric Infrared Sounder ${ }^{3}$ (AIRS), the Tropospheric Emission Spectrometer ${ }^{4}$ (TES), and the Infrared Atmospheric Sounding Interferometer ${ }^{5}$ (IASI), will be in operation.

To achieve the goals mentioned above requires that at least two subtasks be successfully performed. The first one is to design and build such a highspectral-resolution infrared sounder with the re- 
quired signal-to-noise ratio and spectral coverage. The second is to develop an effective retrieval algorithm to invert the observed spectra. In reality, cloud is one of the most difficult obstacles to achieving the second objective. The atmosphere below an optically thick cloud is invisible to a nadir-viewing sounder. Optically thin clouds or patchy clouds also interfere with the retrieval effort. Therefore we must identify cloud signatures from spectra and retrieve relevant quantities from cloudy spectra.

One brute-force approach to the retrieval of cloudy spectra is to retrieve temperature and humidity profiles as well as cloud properties such as cloud top and cloud optical depth simultaneously by using a radiative transfer model including scattering. However, this approach would require extremely large computational power because multiple scattering has to be taken into account. Moreover, retrieving geophysical parameters even from clear-sky spectra is often an ill-posed problem. To include cloud parameters in the retrieval list will make the retrieval problem more complicated and, most likely, increase the nonlinearity of the problem. Therefore, if we could identify cloudy spectra and obtain some a priori information about the clouds from the spectra, the retrieval of cloudy spectra would be facilitated and the computations simplified in those instances when scattering is not effectively present.

A widely used method for detecting and retrieving cirrus from infrared spectra is the trispectral technique.6,7 The basic idea behind this technique is that both ice and liquid water absorb strongly in the infrared window region $(8-12 \mu \mathrm{m})$ but peak at different frequencies. Meanwhile, a weak absorption water-vapor band is also present in the window region. As a result, it is possible to discriminate cirrus, liquid-water clouds and clear sky by the differences in brightness temperature $(\Delta \mathrm{BT})$ between different bands (e.g., $\Delta \mathrm{BT}_{8 \mu \mathrm{m}-11 \mu \mathrm{m}}$ versus $\Delta \mathrm{BT}_{11 \mu \mathrm{m}-12 \mu \mathrm{m}}$ are widely used) in a scatter plot. Also, Hutchison et al. ${ }^{8-10}$ conducted a series of studies to show how to use NOAA's Advanced Very High Resolution Radiometer (AVHRR) and other coincident measurements to detect optically thin cirrus. They used the differences in brightness temperature between AVHRR channel $3(3.7 \mu \mathrm{m})$ and channel 5 $(12 \mu \mathrm{m})$ and information on the total integrated water vapor to detect optically thin cirrus clouds in nighttime AVHRR measurements. ${ }^{8}$ With the help of coincident $1.38-\mu \mathrm{m}$ imagery from the Airborne Visible/Infrared Imaging Spectrometer (AVIRIS), they demonstrated how to detect thin cirrus from daytime AVHRR images over land surfaces. ${ }^{9}$ With imagery from the recent launched AVHRR/3 and information from the HIRS, they also developed an algorithm with which to detect thin cirrus and specific cloud-top phase. ${ }^{10}$ All techniques cited above are narrowband approaches, for which high spectral resolution is not necessary. Moreover, applying these techniques requires measurements at certain spectral ranges. When the resolution is high enough that the individual absorption lines can be resolved, the information content is much greater than that of narrowband measurements. As a result, it might be possible to detect cirrus even when measurements are not made above specific spectral ranges. Several researchers have explored ways in which to extract useful information about clouds from high-spectral-resolution measurements. Turner and Ackerman ${ }^{11}$ explored the possibility of determining cloud phase by use of downwelling brightness temperatures at 9, 12, and $18 \mu \mathrm{m}$ measured with a ground-based Atmospheric Emitted Radiance Interferometer (AERI). Ackerman et al. ${ }^{12}$ also studied cloud retrieval, using upwelling radiance observed by the High-Spectral Resolution Infrared Sounder (HIS). Bantges et al. ${ }^{13}$ used statistical approaches to explore the best possible combinations of spectral ranges for retrieval of the optical properties of cirrus clouds from the nextgeneration spaceborne high-resolution spectrometer such as the IASI. A statistical approach was also used by Schlussel and Goldberg14 to study the retrieval of temperature and humidity profiles from IASI measurements in partly cloudy situations.

In this paper we concentrate on optically thin cirrus clouds (the optical depth of the cloud is $\leq 0.1$, hereafter called thin cirrus clouds). We explore the possibility of using the information in the highresolution spectra to extract some information about cirrus clouds. Cirrus clouds regularly cover $\sim 13 \%$ of the globe. ${ }^{15}$ Therefore the probability of obtaining a spectrum contaminated by cirrus is high. Meanwhile, thin cirrus clouds usually affect the brightness temperature in the window region by only several degrees. As a result, sometimes the effect of thin cirrus is misinterpreted as the effect of surface emission, and this misinterpretation further misleads the retrieval process. So it is meaningful to explore the issue of thin cirrus.

In Section 2 below, we use a simplified three-layer model to demonstrate that a cloud can have different spectral signatures in different absorption lines and to elucidate the physics of the process. In Section 3, by applying a line-by-line radiative transfer model with multiple scattering to standard model atmospheric profiles, we show that the expected spectral signatures for cirrus can also be seen. Sensitivity studies are also presented in Section 3. Additional concerns about the spectral signatures for realistic retrievals are discussed in Section 4. A summary is given in Section 5.

\section{Simplified Analytic Model}

Here we use a simplified model to show what spectral signatures thin cirrus would leave in the residual spectra if thin cirrus spectra were misinterpreted as clear-sky spectra.

Consider a three-layer atmosphere with the planet's surface and the bottom layer at the same temperature $T_{s}$; the temperature for the middle layer is $T_{m}$, and that for the top layer is $T_{T}$. The corresponding blackbody emissions for these three layers are $B_{s}$, $B_{m}$, and $B_{T}$, respectively. We denote the optical depth at the surface $\tau_{0}$, that at the interface between 


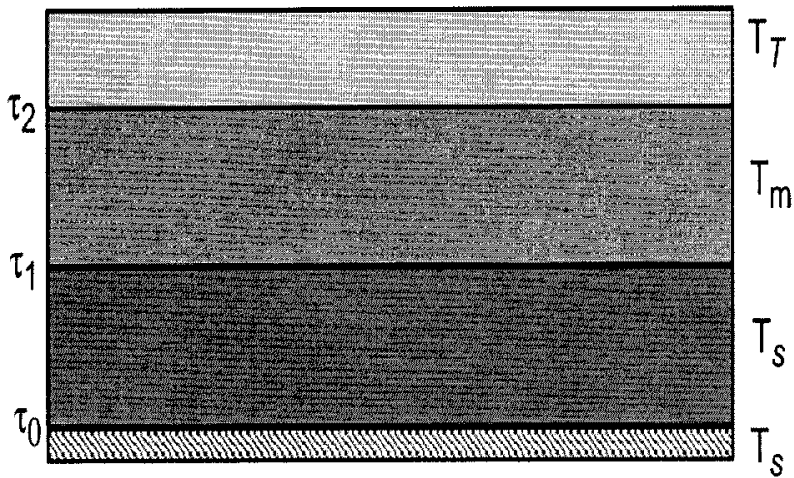

Fig. 1. Schematic of the three-layer atmosphere model. The surface temperature is $T_{s}$. The temperatures of the three layers, $T_{T}$, $T_{m}$, and $T_{s}$, are shown. The optical depth at the interface between the top layer and the middle layer is $\tau_{2}$. The optical depth at the interface between the middle layer and the bottom layer is $\tau_{1}$. The optical depth at the surface is $\tau_{0}$.

the bottom and the middle layer $\tau_{1}$, and that at the interface between the middle and the top layers $\tau_{2}$.

Figure 1 is a schematic plot of this three-layer atmosphere. The radiative transfer equation in a plane-parallel atmosphere in the absence of scattering is given by

$$
\mu \frac{\mathrm{d} I(v)}{\mathrm{d} \tau}=I(v)-B(v),
$$

where $\mu=\cos \theta, \theta$ is the zenith angle, and $B(v)$ is the blackbody emission at frequency $\nu$. Solving Eq. (1) with our three-layer atmosphere configuration for the radiance at the top of atmosphere at zero zenith angle, we obtain

$$
\begin{aligned}
I_{\mathrm{TOA}}(v)= & B_{s}(v) \exp \left[-\tau_{0}(v)\right]-\int_{0}^{\tau_{2}(v)} B_{T}(v) \exp (-\tau) \mathrm{d} \tau \\
& -\int_{\tau_{2}(v)}^{\tau_{1}(v)} B_{m}(v) \exp (-\tau) \mathrm{d} \tau \\
& -\int_{\tau_{1}(v)}^{\tau_{0}(v)} B_{s}(v) \exp (-\tau) \mathrm{d} \tau \\
= & B_{s}(v) \exp \left[-\tau_{1}(v)\right]+B_{m}(v)\left\{\exp \left[-\tau_{2}(v)\right]\right. \\
& \left.-\exp \left[-\tau_{1}(v)\right]\right\}+B_{T}(v)\left\{1-\exp \left[-\tau_{2}(v)\right]\right\} .
\end{aligned}
$$

Also, assuming for analytical simplicity that the mixing ratio of the absorbing gas is uniform in all layers and that the same Lorentzian line shape is applied to all layers, we have

$$
\tau_{2}\left(v-v_{0}\right)=\rho H_{T} S_{T} \frac{\alpha_{L}}{\pi\left[\left(v-v_{0}\right)^{2}+\alpha_{L}^{2}\right]},
$$

where $v_{0}$ is the frequency at the center of the absorption line, $\rho$ is the density of the absorption gas, $H_{T}$ is the vertical thickness of the top layer, $S_{T}$ is the line strength for the top layer, and $\alpha_{L}$ is the half-width of the Lorentzian line shape for the top layer. We define $x=\left(v-v_{0}\right) / \alpha_{L}$ and rewrite Eq. (3) as

$$
\tau_{2}(x)=\frac{C_{2}}{x^{2}+1},
$$

where constant $C_{2}=\rho H_{T} S\left(\alpha_{L} \pi\right)^{-1}$. If we assume that the change in $\alpha_{L}$ from layer to layer is negligible, $\tau_{1}$ can be expressed as

$$
\begin{aligned}
\tau_{1}(x)= & \rho H_{T} S_{T} \frac{\alpha_{L}}{\pi\left[\left(v-v_{0}\right)^{2}+\alpha_{L}^{2}\right]} \\
& +\rho H_{m} S_{m} \frac{\alpha_{L}}{\pi\left[\left(v-v_{0}\right)^{2}+\alpha_{L}^{2}\right]} \\
& =\frac{C_{1}}{x^{2}+1},
\end{aligned}
$$

where $H_{m}$ is the thickness of the middle layer and $S_{m}$ is the line strength for the middle layer. Clearly, we always have $C_{1}>C_{2}$. Similarly, we have $\tau_{0}(x)=$ $C_{0} /\left(x^{2}+1\right)$, where $C_{0}>C_{1}$.

Now we assume that there is a thin cirrus cloud in the middle layer and consider only cloud absorption. For any single absorption line, the cloud's optical depth varies little from the center of line to the wing of the line. Therefore we can assume that the cloud's optical depth $\tau_{c}$ is independent of $x$. Given that the Planck function also varies slowly from the wing to the center of the line, we can also assume that $B_{s}, B_{m}$, and $B_{T}$ do not depend on $x$. The radiance at the top of the atmosphere at zero zenith angle is now

$$
\begin{aligned}
I_{\mathrm{TOA}}(x)= & \exp \left(-\tau_{c}\right)\left\{B_{s} \exp \left(-\frac{C_{1}}{x^{2}+1}\right)\right. \\
& \left.+B_{m}\left[\exp \left(-\frac{C_{2}}{x^{2}+1}\right)-\exp \left(-\frac{C_{1}}{x^{2}+1}\right)\right]\right\} \\
& +B_{T}\left[1-\exp \left(-\frac{C_{2}}{x^{2}+1}\right)\right] .
\end{aligned}
$$

Let us designate the regions that are located at the far wings of any absorption lines and are transparent to the surface emission microwindow regions. In these regions, when cloud is present we have $I_{\mathrm{TOA}}=$ $\exp \left(-\tau_{c}\right) B_{s}$.

Now let us examine a thin cirrus cloud for which $\tau_{c}$ $\ll 1$ holds. If in the retrieval we misinterpret the radiances in microwindow regions as if they were measured in clear-sky conditions, we obtain an incorrect surface emission $B_{s}{ }^{\prime}$ :

$$
B_{s}{ }^{\prime}=B_{s} \exp \left(-\tau_{c}\right)
$$




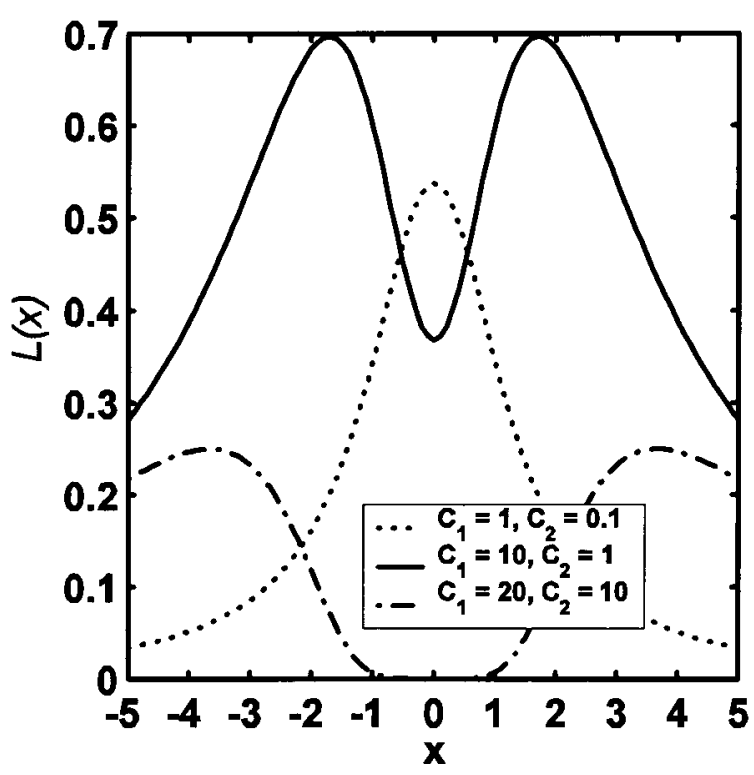

Fig. 2. Plots of $L(x)=\exp \left[-\left(C_{2} / x^{2}+1\right)\right]-\exp \left[-\left(C_{1} / x^{2}+1\right)\right]$ for three combinations of $C_{2}$ and $C_{1}$. Here $x=0$ is the line center and $x=1$ corresponds to the half-width of the Lorentzian line shape. Dotted curve, weak absorption. Solid and dashed-dotted curves, medium and strong absorption, respectively.

Substituting Eq. (7) into Eq. (1) we get

$$
\begin{aligned}
I_{\mathrm{TOA}}{ }^{\prime}(x)= & B_{s} \exp \left(-\tau_{c}\right) \exp \left(-\frac{C_{1}}{x^{2}+1}\right) \\
& +B_{m}\left[\exp \left(-\frac{C_{2}}{x^{2}+1}\right)-\exp \left(-\frac{C_{1}}{x^{2}+1}\right)\right] \\
& +B_{T}\left[1-\exp \left(-\frac{C_{2}}{x^{2}+1}\right)\right] .
\end{aligned}
$$

So the difference between the retrieved radiance [Eq. (8)] and the real radiance [Eq. (6)] is

$$
\begin{aligned}
R(x)= & I_{\mathrm{TOA}}{ }^{\prime}-I_{\mathrm{TOA}} \\
= & {\left[1-\exp \left(-\tau_{c}\right)\right] B_{m}\left[\exp \left(-\frac{C_{2}}{x^{2}+1}\right)\right.} \\
& \left.-\exp \left(-\frac{C_{1}}{x^{2}+1}\right)\right] \propto L(x),
\end{aligned}
$$

where

$$
L(x)=\left[\exp \left(-\frac{C_{2}}{x^{2}+1}\right)-\exp \left(-\frac{C_{1}}{x^{2}+1}\right)\right] .
$$

Figure 2 shows how the shape of $L(x)$ changes with respect to different combinations of $C_{1}$ and $C_{2}$. For weak absorption (Fig. 2, dotted curve), $L(x)$ has one upward-pointing peak centered at the line center. For medium absorption (Fig. 2, solid curve), $L(x)$ has two peaks symmetrical to the line center and one narrow valley. For strong absorption (Fig. 2, dashed-dotted curve), $L(x)$ has a broad valley centered at line center. These results can be under- stood in terms of the weighting functions. ${ }^{16}$ We know that the peak of the weighting function for nadir viewing is located approximately at the altitude where $\tau \approx 1$. So, for weak absorption, the peak of the weighting functions of the line center is in the bottom layer and close to the surface. As a result, when the change in surface temperature has the same effect on the microwindow as the effect of cirrus in the middle layer, the effect of the surface temperature change on absorption at line center is larger than that of the cirrus in the middle layer. So the difference at the center is positive and the difference decreases from center to wing. For strong absorption case, the weighting function of the line center peaks somewhere much higher than the cirrus and the surface. Therefore the absorption at line center is insensitive both to cloud in the middle layer and to surface temperature change, so the difference near the center is almost zero. For medium absorption the weighting function of the line center peaks at an altitude close to the cloud height. Therefore the difference shows a transition shape from the shape of weak absorption to that of strong absorption.

From the above discussion we can show schematically that, if we misinterpret thin cirrus as clear sky, for different absorption lines the shapes of the residuals will be significantly different. When the weighting function peaks at an altitude similar to the cloud height, the residual will show a narrow feature (to which we referred in the abstract as a unique feature). Thus we are led to investigate the possibility of using the residual spectra to detect and obtain some information about thin cirrus and to feed such information back to the retrieval process.

Although the schematic model described above is illustrative of the method, it is too simple for realistic retrieval. In Section 3 we use a much more realistic radiative transfer model and temperature-trace-gas profiles to study this problem.

\section{Line-by-Line Model and Sensitivity Studies}

We use our own line-by-line code for atmospheric radiative transfer with scattering (LBLCARTS) to study the spectral signatures of cirrus clouds in the high-resolution spectra. It is a line-by-line radiative transfer model coupled with the DISORT code ${ }^{17}$ (version 1.3$)^{18}$ for multiple scattering. Sixteen streams are used in DISORT. A Voigt line profile is used in LBLCARTS, and the molecular spectroscopy properties are taken from HITRAN 2K.19 Our LBLCARTS was calibrated against FASCODE 3P (Ref. 20) for the clear-sky calculation. The differences between these two models are less than $1 \%$. As for the cloud, we assume a modified gamma distribution for the cirrus particle size distribution. Then, based on the spectrally dependent refractive index of ice determined by Warren, ${ }^{21}$ we calculate the optical properties of cirrus, using a Mie scattering code. ${ }^{22}$ The temperature profile used in LBLCARTS is taken from the U.S. 1976 Standard Atmosphere profile up to $60 \mathrm{~km}$ (Ref. 23) in 57 layers. For all the calcula- 


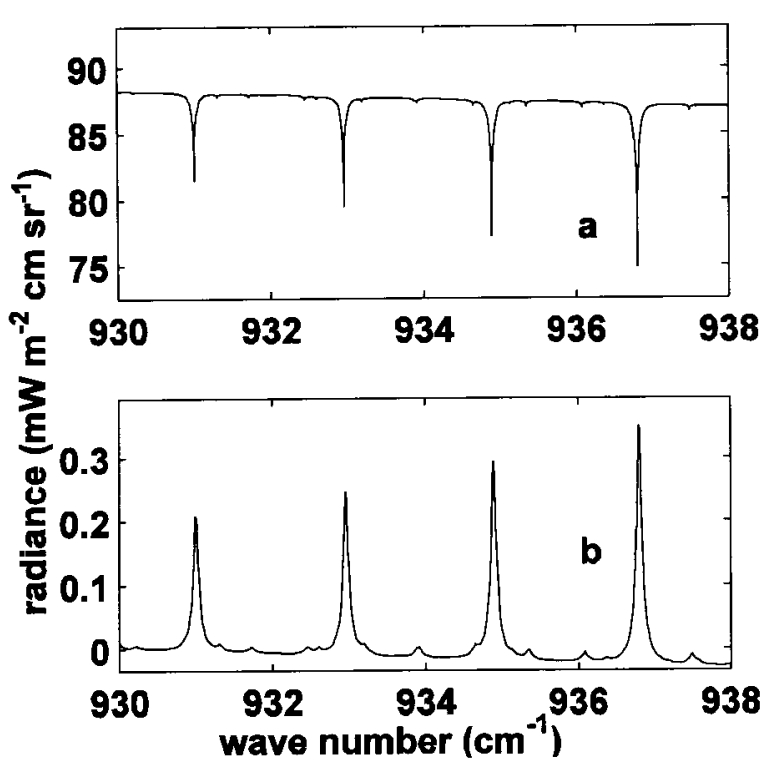

Fig. 3. a, Simulated outgoing spectrum from 930 to $938 \mathrm{~cm}^{-1}$ based on the following configuration: temperature profile of the U.S. 1976 Standard Atmosphere, constant mass mixing ratio of $\mathrm{CO}_{2}, \mathbf{r}_{\mathrm{co} 2}=5.0 \times 10^{-4}$; cirrus cloud with optical depth 0.1 topping at $250 \mathrm{mb}$. The spectrum was calculated from LBLCARTS. b, The difference between the adjusted-surface-temperature spectrum and the cirrus spectrum (hereafter, the residual spectrum) shown in a. Refer to the text for the meaning of the adjustedsurface-temperature spectrum.

tions we assume a surface albedo of 0.02 and a constant $\mathrm{CO}_{2}$ mass mixing ratio of $5.0 \times 10^{-4}$.

Our approach is first to specify a small amount of cirrus cloud at certain levels and calculate the spectra at the top of atmosphere with zero zenith angle. Then we adjust the surface temperature, keep temperatures at other levels unchanged, and repeat the calculation for clear sky. The criterion for adjusting the surface temperature is to let the radiances in the microwindow near $11 \mu \mathrm{m}$ be the same as those derived from the cirrus calculation. Then we take the difference of these two spectra to obtain the residual spectra. Figure 3a is the spectrum at $930-938 \mathrm{~cm}^{-1}$ when thin cirrus with an optical depth of 0.1 is present at $250 \mathrm{hPa}$. There are several weak $\mathrm{CO}_{2}$ absorption lines in this region. Figure $3 \mathrm{~b}$ is the difference between a thin cirrus spectrum and a clearsky spectrum with adjusted surface temperature (hereafter, the residual spectrum) at $930-938 \mathrm{~cm}^{-1}$. It can be seen that after adjustment the baselines of the thin cirrus spectrum and the clear-sky spectrum are the same and the shape of the residual for a weak absorption line is consistent with what we show in Fig. 2 (dotted curve). The solid curve in Fig. 4b shows the residual spectrum at $744-748 \mathrm{~cm}^{-1}$. There are several $\mathrm{CO}_{2}$ medium absorption lines in this region. It can be seen that the narrow feature of the residual for the medium absorption line is also consistent with what we show in Fig. 2 (solid curve).

As we demonstrated in Section 2, the narrow feature is due to the misinterpretation of cirrus, and it is associated with gas medium absorption lines. Therefore it can be expected that under the influence of gas medium absorption the specific angular scattering distribution of thin cirrus should have little effect on the narrow feature. To test this idea we conducted another calculation in which we assumed that the shape of an ice particle is hexagonal instead of spherical. The single-scattering properties of hexagonal ice columns compiled by Baran et al. ${ }^{24}$ were used. The results are marked by circles in Figs. 4a and $4 \mathrm{~b}$. It can be seen from the residual spectra in Fig. $4 \mathrm{~b}$ that the difference between the two shape assumptions is significant only for the region between the two absorption lines. The reason is that gas absorption is weak there, and the thin cirrus has a greater effect on radiance in this region. But, in the line-core region, the difference between hexagonal and spherical particles is small and the amplitudes of the narrow features are almost same. Given this fact, although realistic assumptions about particle shapes should be made in the operational retrieval, for simplicity we still use the spherical particle assumption in the sensitivity study that follows.

To see how these concepts work for nonuniformly mixed gases, we carried out modeling with absorption by methane, which was not uniformly distributed. The results are presented in Figs. 4c and 4d. We used the methane profile from the U.S. 1976 Standard Atmosphere. ${ }^{17}$ Cirrus was still present at 250 $\mathrm{mb}$ with an optical depth of 0.1 . It can be seen that for methane lines whose weighting functions have maximum values near the tropopause, there are similar narrow features in the residual spectra. We can see that, even for a very thin cirrus cloud with an optical depth of 0.1 , the amplitudes of the narrow features in the residual spectra (Figs. 4b and 4d) are $\sim 2 \%$ of the unperturbed radiances (Figs. $4 \mathrm{a}$ and $4 \mathrm{c}$ ). Given the potential signal-to-noise ratio of modern high-resolution infrared sounders, residuals with such amplitudes are expected to be much larger than instrument noise. Therefore this narrow feature in residual spectra can be seen at appropriate absorption lines of different trace gases, and its amplitude is detectable with modern instruments.

In real measurements we can obtain only spectra with limited resolution. It can be imagined that, when resolution is low enough, the narrow feature in the residual spectra will be smoothed out. We investigated this hypothesis by taking the difference after convolving the line-by-line spectra with different hypothetical instrumental line shapes (ILSs) that have different FWHMs. We used a triangle function as an ILS and applied three FWHMs, 0.04, 0.1, and $0.5 \mathrm{~cm}^{-1}$. The results for $\mathrm{CO}_{2}$ lines from 742 to 752 $\mathrm{cm}^{-1}$ are shown in Fig. 5. It can be seen that the narrow features in the residual are clear for FWHM $=0.04 \mathrm{~cm}^{-1}$ and FWHM $=0.1 \mathrm{~cm}^{-1}$. As the FWHM degrades to $0.5 \mathrm{~cm}^{-1}$ the narrow features are barely identified. When we convolved the methane spectra shown in Fig. 4 with the ILSs described above, we reached a similar conclusion. Therefore 


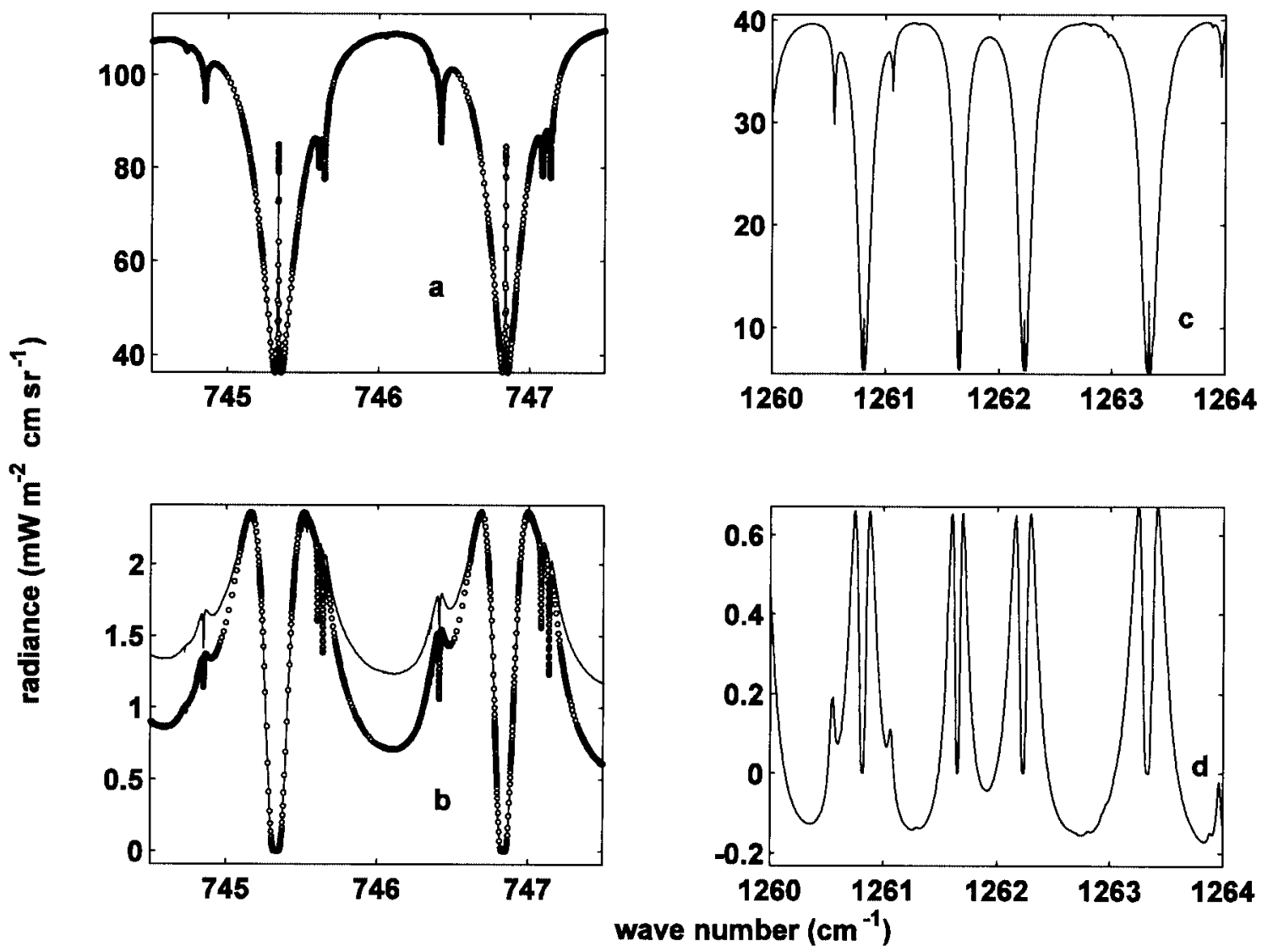

Fig. 4. a, Same as Fig. 3a, except that the region is $744-748 \mathrm{~cm}^{-1}$. For the solid curves the ice particles were assumed to be spherical and the optical properties were computed by Mie scattering theory. For the curves that comprise circles the ice particles were assumed to be hexagonal, and optical properties compiled by Baran et al. ${ }^{24}$ were used. $\quad$ b, Same as Fig. $3 \mathrm{~b}$, except the region is $744-748 \mathrm{~cm}^{-1}$. The solid curve and the circles have same meanings as are in a. c, Simulated outgoing spectrum from 1260 to $1270 \mathrm{~cm}^{-1}$ based on the following configuration: temperature profile of the U.S. 1976 Standard Atmosphere, $\mathrm{CH}_{4}$ profile of the U.S. 1976 Standard Atmosphere, cirrus cloud with optical depth 0.1 topping at $\sim 250 \mathrm{mb}$. (d) Difference between the adjusted-surface-temperature spectrum and the cirrus spectrum shown in c.

the narrow features in the residual are highly sensitive to the instrumental resolution.

For the above discussions the cirrus top was assumed to be at $250 \mathrm{hPa}$ and the optical depth was 0.1 . Next we investigated the sensitivity of the residual spectra to cloud-top height and to the cloud's optical depth. First we changed the cloud-top height to 450 $\mathrm{hPa}$, keeping the optical depth the same. Then we doubled the optical depth but kept the cloud-top height the same. For both cases we calculated the residual spectra according to the method described above (the spectra were all convolved with a triangle function with $0.1-\mathrm{cm}^{-1}$ resolution before the difference was taken). As shown in Fig. 6a, the magnitude of the residual decreased by approximately one half when the cloud-top height was changed from 250 to $450 \mathrm{hPa}$. The narrow features in the residual spectra still can be seen, but these features are wider than their counterparts with the cloud top at 250 $\mathrm{hPa}$. This result is consistent with the discussion in Section 2. As the cloud top was lowered, the features became wider and eventually took the shape of the dashed-dotted curve in Fig. 2. From Fig. 6b,
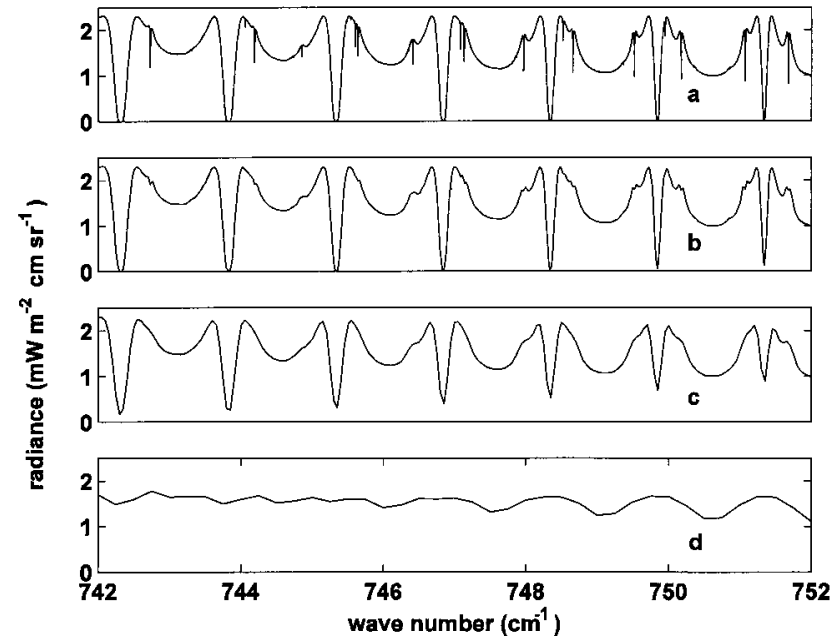

Fig. 5. a, Same as the solid curve in Fig. 4b, except that the region is $742-752 \mathrm{~cm}^{-1}$. b, Convolution of the spectrum in a, and a triangular function with FWHM $=0.04 \mathrm{~cm}^{-1}$. c, Same as $\mathrm{b}$, except that the FWHM is $0.1 \mathrm{~cm}^{-1}$. d, Same as b, except that the FWHM is $0.5 \mathrm{~cm}^{-1}$. 


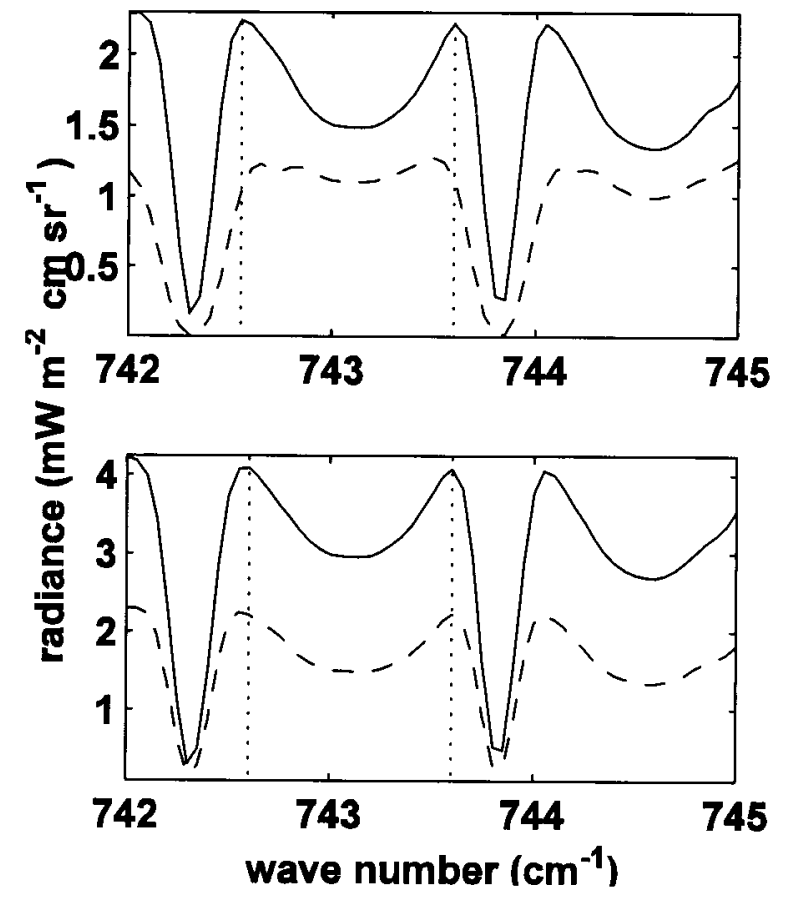

Fig. 6. a, Solid curve, residual spectrum from 742 to $745 \mathrm{~cm}^{-1}$ when the top of the cirrus is at $250 \mathrm{mb}$. Dashed curve, residual spectrum from 742 to $745 \mathrm{~cm}^{-1}$ when the top of the cirrus is at 450 mb. In both cases the cloud's optical depth is 0.1 . The spectra shown here are convolved with a triangular function with a FWHM of $0.1 \mathrm{~cm}^{-1}$. b, Solid curve, residual spectrum from 742 to 745 $\mathrm{cm}^{-1}$ with cloud optical depth $\tau_{\text {cloud }}=0.2$. Dashed curve, the residual spectrum from 742 to $745 \mathrm{~cm}^{-1}$ with cloud optical depth $\tau_{\text {cloud }}=0.1$. For both cases the cloud top is at $250 \mathrm{mb}$. The spectra shown here are convolved with a triangular function with a FWHM of $0.1 \mathrm{~cm}^{-1}$.

when the cloud optical depth was changed from 0.1 to 0.2 the magnitude of the residual was approximately doubled. But the features were as wide as the counterparts of optical depth 0.1. This is so because the cloud top did not change. These results show that the residual spectra can reveal not only the existence of thin cirrus by their narrow features but also information about cloud-top height and optical depth by the magnitude of the residual and the shapes of those narrow features. All these sensitivity studies assumed nadir viewing. If the satellites measure radiance from a nonzero zenith angle, the cirrus optical thickness becomes larger because of the slant path. Therefore, as shown in the simulation, for the same cirrus the amplitudes of the narrow features in the residuals of retrieving nonnadir viewing spectra would be larger than those in the residuals of retrieving nadir-viewing spectra.

We have shown that, in realistic simulations for an appropriate absorption line with the weighting function at line center peaked near the tropopause, the narrow feature that indicates the presence of thin cirrus in the residual spectra can still be seen. The narrow feature is sensitive to the resolution of the ILS, and its magnitude is higher than noise levels of modern high-resolution infrared sounders. The shape and magnitude of the narrow feature are related to cloud-top height and cloud optical depth.

\section{Further Consideration of Realistic Retrieval}

\section{A. Sensitivity Studies}

In Sections 2 and 3 we assumed that misinterpreting the thin cirrus case as a clear-sky case will introduce error only in the estimation of surface temperature. In reality, when we make such a misinterpretation we most often introduce errors into almost all the retrieved quantities. This makes the retrieval problem much more complicated. In this section we explore whether we can see the narrow features in residual spectra in the presence of errors in other retrieved quantities.

We still use our three-layer atmosphere model to explore this problem. But this time we assume that the temperature of the bottom layer is $T_{b}$ and is different from surface temperature $T_{s}$. The corresponding blackbody emission is $B_{b}$. All other configurations are same as those in Section 2. When the cirrus with optical depth $\tau_{c}$ is present in the middle layer, the radiance at the top of atmosphere with zero zenith angle is

$$
\begin{aligned}
I(x)= & \exp \left(-\tau_{c}\right)\left\{B_{s} \exp \left(-\frac{C_{0}}{x^{2}+1}\right)\right. \\
& +B_{b}\left[\exp \left(-\frac{C_{1}}{x^{2}+1}\right)-\exp \left(-\frac{C_{0}}{x^{2}+1}\right)\right] \\
& \left.+B_{m}\left[\exp \left(-\frac{C_{2}}{x^{2}+1}\right)-\exp \left(-\frac{C_{1}}{x^{2}+1}\right)\right]\right\} \\
& +B_{T}\left[1-\exp \left(-\frac{C_{2}}{x^{2}+1}\right)\right] .
\end{aligned}
$$

Now we assume that in the retrieval we misinterpreted thin cirrus as clear sky. To simplify the problem, we assume that the gas concentration is uniform everywhere and that the error in gas-concentration estimation is much smaller than the error in temperature estimation, so we can neglect the former. The retrieved surface emission from the microwindow region is $B_{s} \exp \left(-\tau_{c}\right)$. The retrieved emissions for three layers are $B_{b}+\Delta B_{b}, B_{m}+\Delta B_{m}$, and $B_{T}+\Delta B_{T}$. We define the fractional errors: $\Delta B_{b} / B_{b}=\delta_{b}, \Delta B_{m} /$ $B_{m}=\delta_{m}$, and $\Delta B_{T} / B_{T}=\delta_{T}$. Then the calculated radiance is

$$
\begin{aligned}
I^{\prime}(x)= & \exp \left(-\tau_{c}\right) B_{s} \exp \left(-\frac{C_{0}}{x^{2}+1}\right)+B_{b}\left(1+\delta_{b}\right) \\
& \times\left[\exp \left(-\frac{C_{1}}{x^{2}+1}\right)-\exp \left(-\frac{C_{0}}{x^{2}+1}\right)\right]+B_{m}(1 \\
& \left.+\delta_{m}\right)\left[\exp \left(-\frac{C_{2}}{x^{2}+1}\right)-\exp \left(-\frac{C_{1}}{x^{2}+1}\right)\right] \\
& +B_{T}\left(1+\delta_{T}\right)\left[1-\exp \left(-\frac{C_{2}}{x^{2}+1}\right)\right] .
\end{aligned}
$$


Given $\tau_{c} \ll 1, \exp \left(-\tau_{c}\right) \approx 1-\tau_{c}$. So the residual is approximated by

$$
\begin{aligned}
R(x) \approx & I^{\prime}-I \\
= & B_{b}\left(\delta_{b}+\tau_{c}\right)\left[\exp \left(-\frac{C_{1}}{x^{2}+1}\right)-\exp \left(-\frac{C_{0}}{x^{2}+1}\right)\right] \\
& +B_{m}\left(\delta_{m}+\tau_{c}\right)\left[\exp \left(-\frac{C_{2}}{x^{2}+1}\right)\right. \\
& \left.-\exp \left(-\frac{C_{1}}{x^{2}+1}\right)\right]+B_{T} \delta_{T}[1 \\
& \left.-\exp \left(-\frac{C_{2}}{x^{2}+1}\right)\right] .
\end{aligned}
$$

The last term on the right-hand side of relation (13) is the contribution from the layer above the cirrus. The presence of cirrus has more effect on the retrieval of the temperature profile below the cirrus than above the cirrus. There is also a shortcut approach known as onion peeling, ${ }^{25}$ which can make this term much smaller than the other two terms on the righthand side. For simplicity, we ignore the last term in our discussion. Obviously, if $\left|\delta_{b}\right|\left(\right.$ or $\left.\left|\delta_{m}\right|\right) \gg\left|\tau_{c}\right|$, the signature of the cloud will be smeared out. If $\left|\delta_{b}\right|$ (or $\left.\left|\delta_{m}\right|\right) \ll\left|\tau_{c}\right|$, the signature of the cloud will be dominant. Therefore it is useful for us to estimate the threshold value of the error in temperature estimation leading to $\left|\delta_{b}\right|\left(\right.$ or $\left.\left|\delta_{m}\right|\right) \sim\left|\tau_{c}\right|$. Let us assume that the error in the retrieved temperature is $\Delta T$; when $\Delta T \ll T$ we have

$$
B_{v}(T+\Delta T)=B_{v}(T)+\frac{\partial B_{v}(T)}{\partial T} \Delta T,
$$

where

$$
\begin{aligned}
\frac{\partial B_{v}(T)}{\partial T} & =\frac{\partial}{\partial T}\left\{\frac{2 h v^{3}}{c^{2}[\exp (h v / k T)-1]}\right\} \\
& =B_{v}(T) \frac{\exp (h v / k T)}{\exp (h v / k T)-1} \frac{h v}{k T^{2}} .
\end{aligned}
$$

So, when $\left|\delta_{m}\right| \sim\left|\tau_{c}\right|$, we have

$$
\Delta T=\tau_{c} \frac{k T^{2}}{h v}\left[1-\exp \left(-\frac{h v}{k T}\right)\right] .
$$

If $\tau_{c}=0.1$ for the $\mathrm{CO}_{2}$ lines that we studied in Section $3, v \sim 750 \mathrm{~cm}^{-1}$, and $T \sim 210-290 \mathrm{~K}$, then $\Delta T \sim$ $4.3-8.0 \mathrm{~K}$. This means that, when we perform the retrieval with misinterpretation of thin cirrus, if the error of retrieved temperature profile is smaller than this threshold of $\Delta T$ then the cloud signature will be the dominant contributor to the residual spectra.

To further illustrate this point we used LBLCARTS to carry out a sensitivity analysis. In real satellite remote sensing, one calculates the residual by differencing the measured spectrum and the synthetic spectrum based on retrieved quantities. Here we mimicked this procedure in the following way: Using the 1976 U.S. Standard Atmosphere, we divided the troposphere into four layers; the first was 200$350 \mathrm{hPa}$, the second was $350-500 \mathrm{hPa}$, the third was $500-750 \mathrm{hPa}$, and the last was $750 \mathrm{hPa}$ to the surface. We made two sensitivity studies: (1) For clear sky, we perturbed the temperatures at different layers as follows. For any two neighboring layers the magnitudes of $\Delta T$ are same but the signs of $\Delta T$ are opposite. We call this new temperature profile our retrieved profile. We calculated the two spectra based on the original profile and then on this retrieved profile. Then we took the difference to obtain the residual spectrum. We tried two values of $\Delta T$, namely, 1 and $4 \mathrm{~K}$. (2) For thin cirrus, we changed the temperatures in the same way as for study (1). In addition, the retrieved profile was assumed to be without cirrus and the surface temperature was adjusted according to the description in Section 3. Then we obtained the residual spectrum. The results are shown in Fig. 7. It can be seen that for $\Delta T=1 \mathrm{~K}$ the residuals that are due to the errors in temperature estimation alone are small (Fig. 7a) compared with the error caused by the misinterpretation of cirrus cloud as clear sky (Fig. 7b, solid curve). For $\Delta T=4 \mathrm{~K}$ the effect of errors in temperature estimation on the residual (Fig. 7c) is roughly half of that which is due to the misinterpretation of cirrus cloud (Fig. 7d, solid curve). The residual of case (2) is significantly larger than the residual of case (1). Therefore, if a relation between the retrieved error in temperature and the magnitude of the residual spectrum could be established, in a realistic retrieval it might be possible to determine from the residual spectrum whether thin cirrus is present.

Recently a sensitivity study was made to assess the effect of thin clouds on atmospheric temperature retrievals. ${ }^{26}$ Synthetic spectra were created for a series of cloud optical depths by use of a plane-parallel scattering model. Then a formal retrieval program with no scattering and an assumption of no cloud was used to retrieve atmospheric temperature profiles. When no cloud was present in the synthetic spectra, the temperature errors were $\sim 0.5 \%$ throughout the atmosphere. When clouds were included in the generation of the synthetic spectra but not in the retrieval, temperature errors increased to $\sim 2 \%$ at the surface level for cloud optical depths of as much as 0.05. At larger cloud optical depths (0.15), errors near $2 \%$ were evident at layers between the surface and the cloud height. ${ }^{26}$ In a realistic retrieval, if the errors of estimated temperature profiles are similar to those in this sensitivity study, then, based on our simulation shown in this section, the spectral signature left by cirrus will still be clearly seen in the residual spectra.

\section{B. Analysis of Information Content}

To investigate further how instrumental noise and resolution can affect realistic retrieval in the presence of thin cirrus, we carried out the following study. Using LBLCARTS, we numerically calculated weighting function matrix $\boldsymbol{K}$, defined by $K_{i j}=\partial I_{i}(\boldsymbol{X}) /$ 


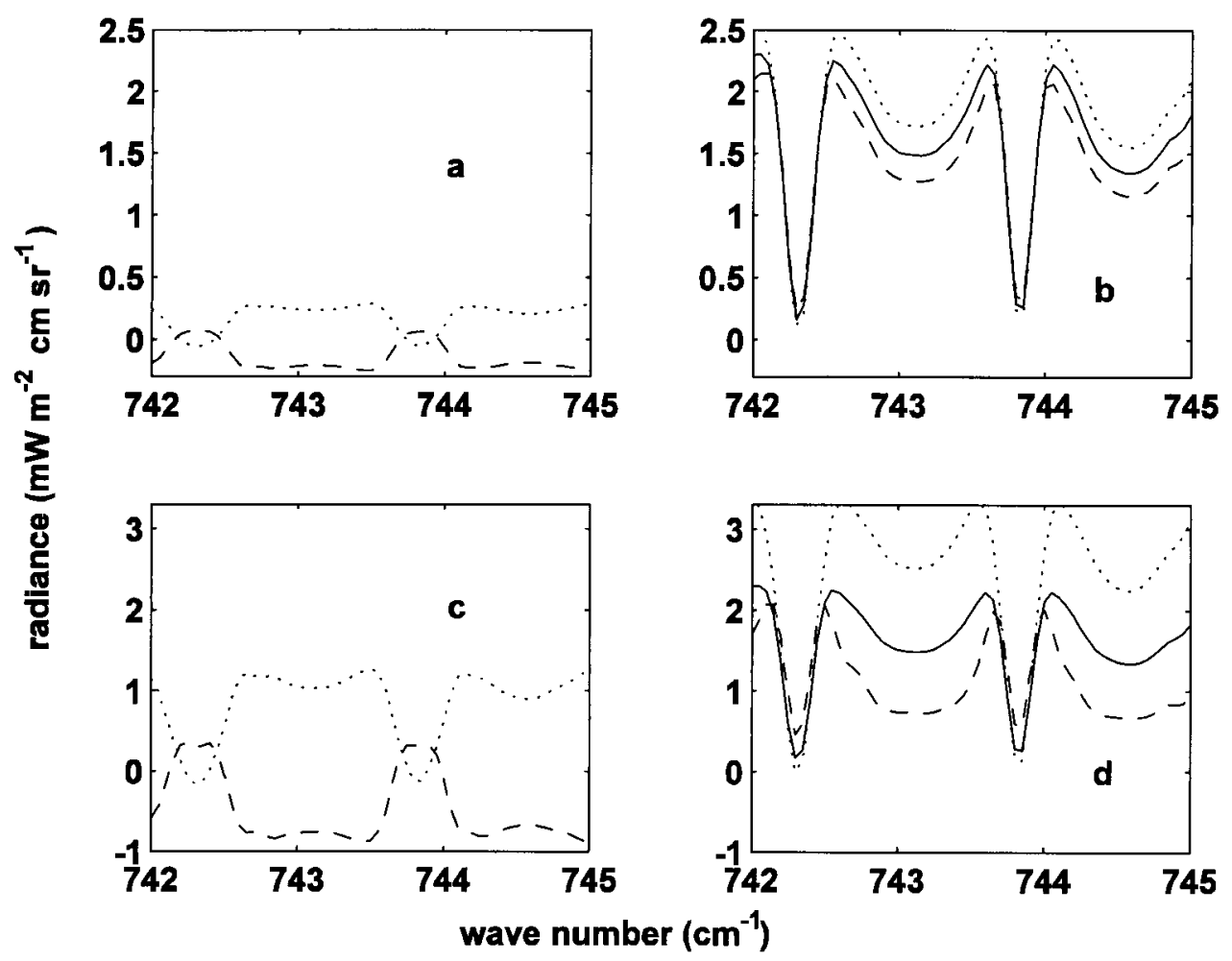

Fig. 7. a, Dashed curve, difference between the retrieved spectrum and the original clear-sky spectrum. The original clear-sky spectrum was calculated based on a 1976 U.S. Standard Atmosphere temperature profile and a constant $\mathrm{CO}_{2}$ mass mixing ratio of $5.0 \times 10^{-4}$. We obtained the retrieved spectrum by changing the temperatures in four layers $(200-350 \mathrm{mb}, 350-500 \mathrm{mb}, 500-750 \mathrm{mb}$, and $750 \mathrm{mb}$ to surface) by $1 \mathrm{~K}$ and $-1 \mathrm{~K}$ alternately. Dotted curve, difference from the retrieved spectrum that we obtained by changing the temperatures in four layers by $-1 \mathrm{~K}$ and $1 \mathrm{~K}$ alternately. The spectra shown here are convolved with a triangular function with a FWHM of $0.1 \mathrm{~cm}^{-1}$. b, Solid curve, difference between the adjusted-surface-temperature spectrum and the cirrus spectrum. Dashed curve, difference between the retrieved spectrum and the cirrus spectrum. We obtained the retrieved spectrum by adjusting the surface temperature as well as changing the temperatures in four layers by $1 \mathrm{~K}$ and $-1 \mathrm{~K}$ alternately. Dotted curve, similar to dashed curve, except that we obtained the retrieved spectrum by changing the temperatures in four layers by $-1 \mathrm{~K}$ and $1 \mathrm{~K}$ alternately. c, Same as a, except that the magnitude of the temperature change is $4 \mathrm{~K}$. d, Same as b, except that the magnitude of the temperature change is $4 \mathrm{~K}$.

$\partial x_{j}$, where $I_{i}(\boldsymbol{X})$ is the radiance at frequency $v_{i}$ and $x_{j}$ is the $j$ th state variable. The state variables were temperatures at 58 levels, concentration of $\mathrm{CO}_{2}$ (for simplicity, a uniform distribution was still assumed for $\mathrm{CO}_{2}$ ), surface albedo, cloud optical depth, and cloud height (for clear sky, the last two quantities were not included). The frequency coverage was $720-760 \mathrm{~cm}^{-1}$ and $910-950 \mathrm{~cm}^{-1}$; one was a strong $\mathrm{CO}_{2}$ absorption band and the other was a weak $\mathrm{CO}_{2}$ absorption band. Also, we created an a priori covariance matrix $\left(\mathbf{S}_{a}\right)$ in a way similar to that of Rodgers. ${ }^{27}$ We also created an error covariance matrix $\left(\mathbf{S}_{\varepsilon}\right)$, assuming that the noise equivalent spectral radiance (NESR) was constant over the selected frequency range and the noise at any frequency outside that range was independent of any other noise. Then we calculated the degree of freedom for the signal $\left(\mathrm{d} f\right.$ ) based on the formula in Rodgers's book ${ }^{28}$ : $\mathrm{d} f=\Sigma_{i} \lambda_{i}{ }^{2} /\left(1+\lambda_{i}{ }^{2}\right)$, where $\lambda_{i}$ is the singular value of matrix $\tilde{\mathbf{K}}=\mathbf{S}_{\varepsilon}{ }^{-1 / 2} \mathbf{K S}_{a}{ }^{1 / 2}$. Here $\mathrm{d} f$ describes the number of useful independent quantities that can be obtained in the measurements with a given error covariance matrix and an a priori covariance matrix. We repeated such calculations several times, varying the NESR and instrumental resolution for both clear sky $\left(\mathrm{d} f_{\text {sky }}\right)$ and thin cirrus $\left(\mathrm{d} f_{\text {cir }}\right)$ and looked for the differences between clear sky and thin cirrus, denoted $\Delta \mathrm{d} f=\mathrm{d} f_{\text {cir }}-\mathrm{d} f_{\text {sky }}$. It could be expected that $\Delta \mathrm{d} f$ would be smaller as the instrumental resolution got coarser, which means that less information about cloud could be retrieved. The results are summarized in Table 1. With an instrumental resolution of $0.03 \mathrm{~cm}^{-1}$ and a NESR smaller than $1 \mathrm{~mW} \mathrm{~m}^{-2} \mathrm{~cm}$ $\mathrm{sr}^{-1}, \Delta \mathrm{d} f$ was 1.5 . This means that, for this resolution and NESR, the thin cirrus case had 1.5 more degrees of freedom than the clear-sky case. Given that the two cases were identical except for the presence of thin cirrus, these 1.5 degrees of freedom were due solely to thin cirrus. The presence of 1.5 degrees of freedom means that at least one and probably two parameters related to thin cirrus could be retrieved. When the resolution was $1.0 \mathrm{~cm}^{-1}, \Delta \mathrm{d} f$ was no more than 1.1 for the three given NESRs. Therefore, for this resolution, we could retrieve at most one quantity (maybe the combination of two parameters) of cirrus.

Table 1 also presents the effect of NESR on the ability to detect cirrus. When NESR increased from 


\begin{tabular}{|c|c|c|c|c|}
\hline \multirow[b]{2}{*}{ FWHM $\left(\mathrm{cm}^{-1}\right)$} & \multirow[b]{2}{*}{$\mathrm{NESR}\left(\mathrm{mW} \mathrm{m}^{-2} \mathrm{~cm} \mathrm{sr}^{-1}\right)$} & \multicolumn{2}{|c|}{ Degree of Freedom } & \multirow[b]{2}{*}{$\Delta \mathrm{d} f=\mathrm{d} f_{\text {cir }}-\mathrm{d} f_{\text {sky }}{ }^{a}$} \\
\hline & & Thin Cirrus $\left(\mathrm{d} f_{\text {cir }}\right)$ & Clear Sky ( $\left.\mathrm{d} f_{\text {sky }}\right)$ & \\
\hline \multirow[t]{3}{*}{0.03} & 0.1 & 20.4 & 18.9 & 1.5 \\
\hline & 1 & 14.0 & 12.5 & 1.5 \\
\hline & 5 & 9.8 & 8.6 & 1.2 \\
\hline \multirow[t]{3}{*}{0.1} & 0.1 & 16.9 & 15.4 & 1.5 \\
\hline & 1 & 11.3 & 9.9 & 1.4 \\
\hline & 5 & 7.6 & 6.6 & 1.0 \\
\hline \multirow[t]{3}{*}{1.0} & 0.1 & 9.9 & 8.8 & 1.1 \\
\hline & 1 & 5.8 & 5.0 & 0.8 \\
\hline & 5 & 3.1 & 2.8 & 0.3 \\
\hline
\end{tabular}

${ }^{a}$ Differences between thin cirrus and corresponding clear sky.

0.1 to $1 \mathrm{~mW} \mathrm{~m}^{-2} \mathrm{~cm} \mathrm{sr}^{-1}$, the difference between thin cirrus and clear-sky $(\Delta \mathrm{d} f)$ changed little, although the degree of freedom decreased. But, when NESR was greater than $1 \mathrm{~mW} \mathrm{~m}{ }^{-2} \mathrm{~cm} \mathrm{sr}^{-1}$, the difference dropped quickly. This result can be understood in term of the cirrus spectral signature discussed above. As long as the NESR was considerably smaller than the magnitude of the cirrus signature, it did not smear out the thin cirrus information in the spectra. Therefore, although the degree of freedom $(\mathrm{d} f$ cir and $\mathrm{d} f_{\text {sky }}$ ) decreased as the NESR became larger, the difference between thin cirrus and clear-sky $(\Delta \mathrm{d} f)$ was essentially unchanged. But when NESR was larger than the magnitude of the cirrus signature, it would smear out the thin cirrus information. As a result, the difference $(\Delta \mathrm{d} f)$, as well as the degree of freedom $\left(\mathrm{d} f_{\text {cir }}\right.$ and $\left.\mathrm{d} f_{\text {sky }}\right)$, decreased when NESR increased.

The above analysis shows how the degrees of freedom for cirrus decrease when instrumental resolution is degraded. The degrees of freedom related to thin cirrus remain the same as long as the NESR is smaller than the magnitude of the cirrus spectral signature. This result is consistent with our sensitivity analysis.

\section{Summary}

In this paper we explored methods for using the information from high-spectral-resolution measurements to help retrieval in the presence of thin cirrus clouds. With the simple analytic model and LBLCARTS, we demonstrated that, when thin cirrus is misinterpreted as surface emission under clear-sky situation in the retrieval, the shapes of the residuals are different for absorption lines with different absorptivities. Those lines with weighting functions peaking about the tropopause show narrow features in the residual spectrum. The residual is much higher than the potential instrumental noise levels of modern high-resolution infrared sounders. This holds for both uniformly distributed gases such as carbon dioxide and nonuniformly distributed gases such as methane. These narrow features are sensitive to the resolution of the ILS. For thin cirrus with optical depth 0.1 , these features can be clearly seen with a resolution of the ILS of $0.1 \mathrm{~cm}^{-1}$. When the resolution of the ILS is $0.5 \mathrm{~cm}^{-1}$, these features cannot be seen at all. These features are also sensitive to cloud height and cloud optical depth. In reality, the issue is more complicated because the errors in other retrieved quantities also contribute to the residual spectrum. Our sensitivity simulations show that when the errors in temperature estimation are not large, the effect of the misinterpretation of cirrus is still dominant in the residual. The analysis presented in Subsection 4.B is also consistent with our sensitivity analysis.

To retrieve standard geophysical parameters in the presence of thin cirrus is a complicated problem. Here we looked at this problem from the point of view of high-spectral-resolution measurement. We conducted some sensitivity analyses to explore how the high-resolution information can provide additional clues for the retrieval. Realistic retrievals will follow this procedure: First apply the clear-sky retrieval routine to a spectrum and then evaluate the magnitude and the shape of residual spectrum to decide whether thin cirrus is present. If cirrus is present, try to make an estimate of the cloud's optical depth and the cloud-top height based on the residual and then feed these estimates as initial guesses to the cirrus retrieval routine to retrieve the spectrum. Our future studies will incorporate the ideas presented here into an operational retrieval algorithm.

We thank M. Gerstell and Z. M. Kuang for valuable comments. We also thank two anonymous referees for improving the paper. This research is supported by NOAA grant NA06EC0505 to the California Institute of Technology.

\section{References}

1. W. L. Smith, H. M. Woolf, C. M. Hayden, D. Q. Wark, and L. M. Mcmillin, "TIROS-N operational vertical sounder," Bull. Am. Meteorol. Soc. 60, 1177-1187 (1979).

2. World Meteorological Organization, The World Weather Watch Programme 1988-1997 (World Meteorological Organization, Geneva, 1987).

3. H. Aumann and R. Pagano, "Atmospheric Infrared Sounder on the Earth Observing System,” Opt. Eng. 33, 776-784 (1994).

4. R. Beer, T. Glavich, and D. Rider, "Tropospheric emission spec- 
trometer for the Earth Observing System's Aura satellite," Appl. Opt. 40, 2356-2367 (2001).

5. D. Diebel, M. Langevin, D. Klaes, P. Courtier, T. Phulpin, F. Cayla, and G. Chalon, "The advanced atmospheric temperature sounder IASI-a new development for the polar satellite Metop," presented at the 1997 Meteorological Satellite Data Users' Conference, Brussels, Belgium, 29 September-3 October 1997 (European Organization for the Exploitation of Meteorological Satellites, Darmstadt, Germany).

6. S. A. Ackerman, W. L. Smith, J. D. Spinhirne, and H. E. Revercomb, "The 27-28 October 1986 FIRE IFO cirrus case study: spectral properties of cirrus clouds in the $8-12-\mu \mathrm{m}$ window," Mon. Weather Rev. 118, 2377-2388 (1990).

7. Y. Takano, K. N. Liou, and P. Minnis, "The effects of small ice crystals on cirrus infrared radiative properties," J. Atmos. Sci. 49, 1487-1493 (1992).

8. K. D. Hutchison, K. R. Hardy, and B. C. Gao, "Improved detection of optically thin cirrus clouds in nighttime multispectral meteorological satellite imagery using total integrated water-vapor information,” J. Appl. Meteorol. 34, 1161-1168 (1995).

9. K. D. Hutchison and N. J. Choe, "Application of $1.38 \mu \mathrm{m}$ imagery for thin cirrus detection in daytime imagery collected over land surfaces," Int. J. Remote Sens. 17, 3325-3342 (1996).

10. K. D. Hutchison, "Application of AVHRR/3 imagery for the improved detection of thin cirrus clouds and specification of cloud-top phase," J. Atmos. Ocean. Tech. 16, 1885-1899 (1999).

11. D. D. Turner and S. A. Ackerman, "Cloud phase and microphysical property retrieval using the atmospheric emitted radiance interferometer (AERI)" in 11th Conference on Atmospheric Radiation (American Meteorological Society, Boston, Mass., 2002), p. 60.

12. S. A. Ackerman, H. E. Revercomb, R. O. Knuteson, and P. Antonelli, "Analysis of the high-spectral resolution infrared sounder (HIS) radiances collected as part of the FIRE program," in 11th Conference on Atmospheric Radiation (American Meteorological Society, Dallas, Texas, 2002), p. 245.

13. R. J. Bantges, J. E. Russell, and J. D. Haigh, "Cirrus cloud top-of-atmosphere radiance spectra in the thermal infrared," J. Quant. Spectrosc. Radiat. Transfer 63, 487-498 (1999).

14. P. Schlussel and M. Goldberg, "Retrieval of atmospheric temperature and water vapour from IASI measurements in partly cloudy situations," Adv. Space Res. 29, 1703-1706 (2002).

15. W. B. Rossow and R. A. Schiffer, "Advances in understanding clouds from ISCCP,” Bull. Am. Meteorol. Soc. 80, 2261-2287 (1999).
16. R. M. Goody and Y. L. Yung, Atmospheric Radiation: Theoretical Basis (Oxford U. Press, New York, 1989).

17. K. Stamnes, S.-C. Tsay, W. Wiscombe, and K. Jayaweera, "Numerically stable algorithm for discrete-ordinate-method radiative transfer in multiple scattering and emitting layered media," Appl. Opt. 27, 2502-2509 (1988).

18. DISORT version 1.3 was released in March 2000 and can be obtained from ftp://climate.gsfc.nasa.gov/pub/wiscombe/ Multiple_Scatt/.

19. L. S. Rothman, C. P. Rinsland, A. Goldman, S. T. Massie, D. P. Edwards, J. M. Flaud, A. Perrin, C. Camy-Peyret, V. Dana, J. Y. Mandin, J. Schroeder, A. McCann, R. R. Gamache, R. B. Wattson, K. Yoshino, K. V. Chance, K. W. Jucks, L. R. Brown, V. Nemtchinov, P. Varanasi, "The HITRAN molecular spectroscopic database and HAWKS (HITRAN Atmospheric Workstation): 1996 edition,” J. Quant. Spectrosc. Radiat. Transfer 60, 665-710 (1998).

20. J. Wang and G. P. Anderson, "Validation of FASCOD3 and MODTRAN3: comparison of model calculations with interferometer observations from SPECTRE and ITRA," Appl. Opt. 35, 6028-6040 (1996).

21. S. G. Warren, "Optical-constants of ice from the ultraviolet to the microwave," Appl. Opt. 23, 1206-1225 (1984).

22. J. E. Hansen and L. D. Travis, "Light scattering in planetary atmospheres," Space Sci. Rev. 16, 527-610 (1974).

23. G. P. Anderson, S. A. Clough, F. X. Kneizys, J. H. Chetwynd, and E. P. Shettle, "AFGL atmospheric constituent profiles $(0-$ 120 km), Rep. AFGL-TR_86-0110 (U.S. Air Force Geophysics Laboratory, Hanscom Air Force Base, Mass., 1986).

24. A. J. Baran, S. Havemann, and D. Mackowski, "A database of hexagonal column optical properties for wavelengths ranging between $0.2 \mathrm{~mm}$ to $30 \mathrm{~mm}$ produced for ANNEX 7," contract 4b/3/02 (Department of the Environment, Food, and Rural Affairs, London, UK (2002).

25. J. M. Russell and S. R. Drayson, "The inference of atmospheric ozone using satellite horizon measurements in the $1042 \mathrm{~cm}^{-1}$ band," J. Atmos. Sci. 29, 376-390 (1972).

26. A. Eldering, Jet Propulsion Laboratory, Mail Stop 183-601, 4800 Oak Grove Drive, Pasadena, Calif. 91109 (personal communication, 2002).

27. C. D. Rodgers, Inverse Methods for Atmospheric Sounding: Theory and Practice (World Scientific, Singapore, 2000), Chap. 2, pp. 37-41.

28. Ref. 27 , p. 37 , formula (2.80). 